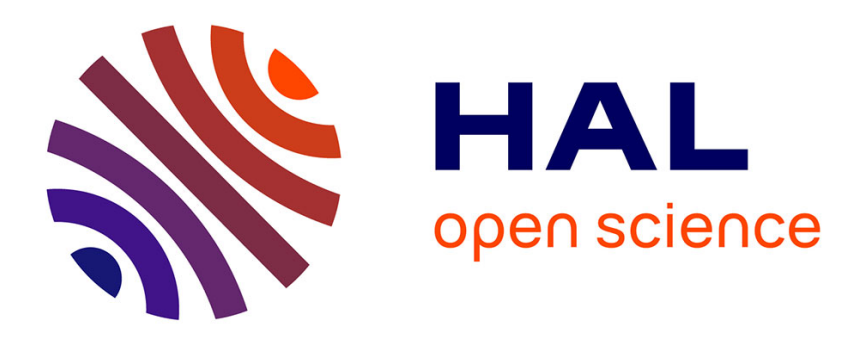

\title{
Acoustique des cavités : modèles et applications
}

M. Bruneau

\section{- To cite this version:}

M. Bruneau. Acoustique des cavités : modèles et applications. Journal de Physique IV Proceedings, 1994, 04 (C5), pp.C5-675-C5-684. 10.1051/jp4:19945145 . jpa-00252823

\section{HAL Id: jpa-00252823 https://hal.science/jpa-00252823}

Submitted on 1 Jan 1994

HAL is a multi-disciplinary open access archive for the deposit and dissemination of scientific research documents, whether they are published or not. The documents may come from teaching and research institutions in France or abroad, or from public or private research centers.
L'archive ouverte pluridisciplinaire HAL, est destinée au dépôt et à la diffusion de documents scientifiques de niveau recherche, publiés ou non, émanant des établissements d'enseignement et de recherche français ou étrangers, des laboratoires publics ou privés. 


\title{
Acoustique des cavités : modèles et applications
}

\author{
M. BRUNEAU
}

Laboratoire d'Acoustique de l'Université du Maine, Associé au CNRS, Université du Maine, Avenue Olivier Messiaen, BP. 535, 72017 Le Mans cedex, France

\begin{abstract}
Résumé : The purpose of this paper is to present the main developments in the theory of the acoustics of dissipative fluids at rest in bounded spaces (principally cavities of different sizes and shapes ), from the early beginnings ( in the 1850' s) until now, including a brief indication of possible approaches for future developments. Several applications of interest, and current research concerned with miniaturisation, are presented, with emphasis being given to the work done on the acoustic gyrometer and on its potential to be miniaturized, which include the miniaturization of acoustical transducers (on silicon chips).
\end{abstract}

\section{INTRODUCTION.}

L'objectif principal de cet exposé est de présenter les développements théoriques qui visent à proposer une description précise, et au demeurant relativement aisée à manipuler, du comportement dynamique des fluides compressibles, visqueux, conducteurs de la chaleur (et éventuellement siège de relaxation moléculaire), au repos, dans le cadre de l'acoustique linéaire. L'exposé porte plus particulièrement sur les espaces clos, les cavités miniatures et les fines couches de fluide.

Depuis plus de cent ans, des descriptions précises de la propagation dans les tubes, qui prennent en compte les effets dissipatifs, sont rendues nécessaires du fait des exigences des applications de la propagation guidée ( $\mathrm{y}$ compris en tube capillaire). Par ailleurs, depuis le début des années 1930, l'étude des champs acoustiques dans les petites cavités, nécessaire en particulier pour la réalisation et l'étalonnage des transducteurs $[1,2,3,4,5,6]$, connaît des développements significatifs, et plus particulièrement depuis les années 1970 qui furent marquées dans le domaine par la mesure des propriétés des gaz à partir des mesures précises de la vitesse du son, sur la base de modèles appropriés. Plus récemment, une attention particulière est portée sur des applications nouvelles telles la gyrométrie acoustique, l'amortissement de vibrations par de fines couches de fluide, l'optimisation des performances des transducteurs, etc. Enfin, le besoin de modèles précis pour les champs acoustiques en cavités de plus en plus petites se fait jour actuellement, incluant les très minces couches de fluides, en raison du développement des transducteurs miniaturisés et du large usage attendu des technologies sur silicium pour ce type d'applications.

L'exposé est divisé en deux partie: - dans la première la théorie de la propagation en fluide dissipatif est rappelée, sous forme d'abord d'un bref rappel historique, puis d'une discussion plus approfondie des résultats obtenus ces dix dernières années, - dans la seconde partie des exemples d'applications sont présentés, et plus particulièrement ceux qui nécessitent des développements théoriques assez précis; ceux qui sont rendus nécessaires par la miniaturisation des transducteurs et par de nouvelles applications font enfin l'objet d'un exposé qui reste ouvert de nos jours. 


\section{EVOLUTION DES EQUATIONS DE L'ACOUSTIQUE EN FLUIDE DISSIPATIF.}

\subsection{Premières études sur les phénomènes dissipatifs dans les fluides (1820 - 1900).}

La dissipation de l'énergie des ondes acoustiques en milieu fluide relève essentiellement de trois processus distincts : - les transferts d'énergie par effets de viscosité, - les transferts de chaleur par conduction thermique, - les transferts d'énergie des mouvements translationnels des molécules vers les modes internes (et inversement) qui résultent des collisions intermoléculaires et qui dans certaines circonstances font apparaître des retards non négligeables à l'accès à l'état d'équilibre.

Datant des années 1820 , la théorie de la propagation en fluide dissipatif reste relativement récente. Les premiers apports furent ceux qui portent sur la diffusion de la chaleur des régions de température élevée (associée aux compressions en acoustique) vers celles de plus basse température (associées aux détentes en acoustique). Jean B.J. Fourier (1768-1830) s'est penché sur ce problème au tout début du 19ème siècle, mais ce n'est qu'en 1822 qu'il paracheva sa réflexion sur les flux de chaleur dans un ouvrage intitulé "Analytic Theory of Heat" (à noter qu'à la même époque il découvrit le théorème fameux qui porte son nom).

La seconde découverte d'importance sur les phénomènes dissipatifs est celle de Sir George Stokes qui porte sur les effets de la viscosité de cisaillement (1845), et qui permet de formuler ces effets de dissipation dans les équations fondamentales de la dynamique. Stokes (1819-1903) étudia le comportement des fluides visqueux de 1845 à 1850 . Il énonça ce que nous appelons aujourd'hui la loi de Stokes qui s'applique au mouvement de petites sphères dans un fluide visqueux sous l'action des forces de gravité. (Il travailla de surcroît sur la fluorescence, le son, et sur la lumière; il fût parmi ceux qui suggérèrent les premiers en 1896 que les rayons X, découverts par Röentgen, sont des rayonnements électromagnétiques qui s'apparentent à la lumière.)

Mais c'est la fameuse publication de Kirchhoff en 1868 [7], portant sur les effets de dissipation visqueuse et thermique en acoustique, qui marque le début de la théorie moderne de la propagation acoustique en fluides visqueux et conducteurs de la chaleur. Gustav R. Kirchhoff est né le 12 mars 1824 et est décédé le 17 octobre 1887 . Sa contribution à la physique la plus célèbre reste néanmoins la découverte expérimentale et la formulation théorique de la loi fondamentale du rayonnement électromagnétique (1859), et l'introduction un peu plus tard (1862) du concept de corps noir. Ses autres travaux célèbres portèrent sur les courants électriques (18451849) et sur la propagation de l'électricité dans les conducteurs (1857). Kirchhoff avait le génie de formuler les phénomènes physiques sous forme de concepts logiques qui conduisent à des systèmes cohérents dégagés d'éléments de nature hypothétique; c'est ainsi qu'en 1868 il donna une description très fertile de la propagation sonore dans les gaz, mentionnée ci-dessus. Cette théorie repose principalement sur les équations linéarisées de Stokes-Navier, qui prennent en compte les effets de viscosité de cisaillement et de volume, et sur celle de Fourier pour la conduction thermique. Il obtint une équation de dispersion dont la solution lui permit d'exprimer les constantes de propagation pour les ondes planes et les ondes sphériques en espace infini, et pour les ondes planes qui se propagent dans un tube circulaire. Dans ce dernier cas, il imposa des conditions de vitesse nulle et de variation de température nulle en frontière (sur les parois du tube). Il en déduisit, dans le cas des tubes "larges" et des "grandes" longueurs d'onde, le facteur d'atténuation et la vitesse de propagation des ondes planes, ne conservant dans les calculs que les termes d'ordre le plus bas...

Lord Rayleigh traite de ces problèmes en détail dans son ouvrage fameux "Theory of Sound" [8]. Prix Nobel de physique en 1904, Lord Rayleigh (baron J.W.Strutt, 1842-1919) a publié de nombreux documents sur ses travaux théoriques et expérimentaux, qui portent sur les rayonnements optiques et acoustiques, l'électromagnétisme, les théorèmes généraux de la mécanique, les vibrations des milieux élastiques, la capillarité et la thermodynamique. Une illustration de sa surprenante capacité à pressentir des phénomènes physiques à l'origine de résultats d'observations nous est donnée dans une de ses puplications (1899) intitulée "On the cooling of air by radiation and conduction and on the propagation of sound". Dans ce document; il pose le problème de l'atténuation acoustique anormalement élevée dans l'air ( nettement plus élevée que celle prédite par les propriétés de transports liées à la viscosité et à la conduction thermique ). Il suggéra de chercher l'explication à ces écarts systématiques dans des mécanismes de relaxation associés aux transferts d'énergie entre mouvements de translation et mouvements internes des molécules du gaz, sous l'effet du passage de l'onde sonore. Cette hypothèse fut 
retenue ultérieurement par de nombreux chercheurs et conduisit au développement de l'acoustique moléculaire qui, au cours de la seconde partie du vingtième siècle, a connu de nouvelles et importantes applications, non seulement dans le domaine de la propagation ultrasonore, mais encore dans ceux liés à l'étude de la structure des molécules et de leurs interactions [9].

Ainsi, à la fin du siècle dernier, les idées et équations de base étaient introduites. Elles permettaient d'interpréter les mécanismes de dissipation dits classiques (c'est-à-dire ceux dus aux effets de viscosités et de conduction thermique), et de comprendre les mécanismes de relaxation moleculaire. Une interprétation complète de l'absorption du son doit a priori non seulement prendre en compte la contribution de chaque mécanisme pris séparément, mais encore leurs interactions. Heureusement, pour les fréquences inférieures à $10 \mathrm{MHz}$, l'absorption classique et celle due à la relaxation moléculaire sont additives dans les gaz. Ce résultat fut admis sans démonstration pendant longtemps; il a été démontré assez récemment (1972) [10] à partir de solutions approchées de l'équation de Boltzmann. De plus, comme nous le voyons dans la suite, dans les guides d'onde et les cavités auxquelles nous sommes ici intéressés, la dissipation à prendre en compte provient des fines couches limites en parois, liées à la viscosité et à la conduction thermique. Par suite, dans les conditions usuelles, la relaxation moléculaire peut être négligée dans les processus de dissipation. Cependant, comme nous allons le voir dans les équations fondamentales, les effets de la relaxation moléculaire peuvent être pris en compte en écrivant simplement que la capacité calorifique du gaz à pression constante est un nombre complexe qui dépend d'un temps de relaxation, sans changer quoique ce soit d'autre dans la solution formelle du problème. C'est ainsi que, du fait que cet exposé porte essentiellement sur la propagation acoustique en petits espaces clos, seuls les processus de dissipation dus à la viscosité et à la conduction thermique sont à prendre en compte. Les travaux de Kirchhoff sur la propagation acoustique apparaissent donc comme ceux sur lesquels repose la plupart des travaux ultérieurs qui portent sur les champs acoustiques en fluides visco-thermiques confinés en espaces limités.

\subsection{Etudes sur la propagation en fluide visco-thermique (1900 à 1980).}

La première moitié du vingtième siècle s'est écoulée sans qu'aucun modèle ne fournisse de solution aux problèmes tels que celui de la propagation des modes supérieurs dans les guides, qu'ils soient propagatifs ou évanescents. Ce n'est seulement que 80 années après la fameuse publication de Kirchhoff que Lothar Cremer a publié un article (1948) intitulé "Sur les couches limites acoustiques au voisinage d'une paroi rigide" [11] dans lequelle il montre que, lors de la réflexion d'une onde plane harmonique sur une paroi plane infinie rigide, le comportement acoustique du milieu, au voisinage de la paroi mais hors de la couche limite, est décrit de manière convenable par un simple mode acoustique, et que la perte d'énergie provient exclusivement des effets de viscosité et de conduction thermique à l'intérieur de la couche limite. Par suite, écrivant que la variation de température et la vitesse particulaire sont nulles sur la paroi, la réflexion d'une onde plane sur une surface plane peut être décrite par le rapport de la composante normale de la vitesse particulaire acoustique à la pression acoustique à la frontière, appelé "admittance spécifique apparente", qui dépend du coefficient de viscosité de cisaillement, du coefficient de conduction thermique et du rapport du nombre d'onde acoustique à sa composante normale au plan frontière (c'est-à-dire l'angle d'incidence pour les modes propagatifs). C'est un résultat très utile, dont une démonstration est proposée au paragraphe suivant. Il fût utilisé deux ans après sa publication (1950) pour calculer l'atténuation des modes propagatifs supérieurs dans les tubes [12] , puis assez récemment pour calculer l'atténuation des modes évanescents (dès la coupure adiabatique) [13 à 16$]$.

La théorie de Kirchhoff et le modèle de Cremer furent également utilisés récemment dans le calcul des champs acoustiques ( fréquences de résonances, facteurs de qualité, ...) en cavités rectangulaires, cylindriques et sphériques, en vue d'application à la mesure précise de la célérité du son dans les gaz [17], à la gyrométrie acoustique, ... . Mais, des exigences nouvelles apparaissent aujourd'hui, issuent de travaux récents sur les minces couches de fluides ou sur les fluides en cavités miniatures, pour lesquelles le besoin de modèles précis adaptés amène à rechercher des solutions des équations de base qui n'admettent plus les hypothèses et approximations d'usage courant . Le paragraphe suivant a pour objet de présenter ces équations de base, puis des formes de solutions et l'équation de dispersion générale en présence de frontières, et enfin quelques résultats récents relevant de l'acoustique des cavités. 

2.3 Développements récents (1980...) sur le comportement acoustique des fluides dissipatifs
en espaces bornés.

\subsubsection{Les équations de base.}

La propagation en fluide visco-thermique peut être décrite par un ensemble de paramètres thermostatiques et un ensemble de variables thermodynamiques. Les paramètres thermostatiques essentiels sont les valeurs moyennes de la pression $\mathrm{P}$, de la température $\mathrm{T}$ et de la masse volumique $p$. Les variables thermodynamiques comportent les variations de la pression $p$, de la température $\tau$, de la masse volumique $\rho$ ' et de l'entropie $s$, et la vitesse particulaire $\mathbf{v}$. Les propriétés du fluide font intervenir les quantités "phénoménologiques" suivantes : les coefficients de viscosité de cisaillement $\mu$ et de volume $\eta$, le coefficient de conduction thermique $\lambda$, les capacités calorifiques par unité de masse à pression constante $C_{p}$ et à volume constant $C_{V}$, leur rapport $\gamma$, le coefficient d'augmentation de pression à volume constant $\left.\beta=\partial_{\mathrm{T}} \mathrm{P}\right)_{V}$, et le coefficient de compressibilité isotherme $\chi_{\mathrm{T}}$. Trois sortes de sources peuvent être à l'origine du mouvement : forces $\mathbf{F}$, débit $q$ et flux de chaleur $r$ ( ces trois quantités étant prises par unité de masse).

Le système complet d'équations de base linéarisées pour la propagation comporte[1 18 à 21$]$ :

-L'équation de Navier-Stokes:

$$
\rho \partial_{t} v+\operatorname{grad} p-(\eta+4 \mu / 3) \operatorname{grad}(\operatorname{div} v)+\mu \operatorname{rot} \operatorname{rotv}=\rho \mathbf{F}
$$

-L'équation de conservation de la masse:

$$
\partial_{t} \rho^{\prime}+\operatorname{div}(\rho \mathbf{v})=\rho q \text {, }
$$

-L'équation de conservation de l'énergie, réduite à:

$$
\rho T \partial_{t} s-\operatorname{div}(\lambda \operatorname{grad} T)=\rho r .
$$

De plus, les lois de la thermodynamique permettent d'exprimer toute variable d'état en fonction de deux d'entre elles (milieux bi-variants), ce qui permet d'écrire par exemple:

$$
\begin{aligned}
& s=\left(C_{\mathrm{p}} / T\right) \tau-\left(P \beta \chi_{\mathrm{T}} / \rho\right) p, \\
& \rho^{\prime}=\rho \chi_{\mathrm{T}}(p-P \beta \tau)
\end{aligned}
$$

La relaxation moléculaire peut ici être prise en compte en écrivant simplement que la capacité calorifique $C_{p}$ dans l'équation (4) est un nombre complexe $C_{p}{ }^{*}$ qui dépend du temps de relaxation $\theta$; pour les gaz bi-atomiques $\mathrm{C}_{\mathrm{p}}{ }^{*}$ est donné par :

$$
\mathrm{C}_{\mathrm{p}}{ }^{*}=\mathrm{C}_{\mathrm{p}}-\mathrm{C}_{\mathrm{v}}(\mathrm{v}) \theta \partial_{\mathrm{t}} /\left(1+\theta \partial_{\mathrm{t}}\right) \text {, }
$$

où $C_{V}{ }^{(v)}$ est la part due à la vibration des molécules qui contribue à la valeur de la capacité calorifique à volume constant, et où l'opérateur $\left(1+\theta \partial_{t}\right)^{-1}$ signifie formellement

$$
\theta^{-1} \mathrm{e}^{-\mathrm{t} / \theta} \int \mathrm{dt}^{\prime} \mathrm{e}^{\mathrm{t}^{\prime} / \theta}
$$

Mais pour les guides d'onde et les cavités considérés ici, l'énergie se trouve dissipée en quasi-totalité dans les couches limites visqueuses et thermiques, et par suite les effets dissipatifs dus à la relaxation moléculaire restent négligeables (on écrit $C_{p}{ }^{*}=C_{p}$ ).

La vitesse particulaire $\mathbf{v}$ de toute perturbation qui relève de ces équations peut être considerée comme la superposition d'une vitesse indivergente $\mathbf{v}_{\mathrm{v}}$ (associée aux effets de viscosité) et d'une vitesse irrotationnelle $\mathbf{v} l$, associée aux effets acoustiques ( $\mathbf{v a}_{\mathbf{a}}$ ) et à ceux de conduction thermique $\left(\mathbf{v}_{\mathrm{h}}\right)$ :

$$
\mathbf{v}=\mathbf{v}_{l}+\mathbf{v}_{\mathrm{v}} \quad, \quad \mathbf{v} l=\mathbf{v}_{\mathrm{a}}+\mathbf{v}_{\mathrm{h}}
$$

Par suite, l'équation (1) peut être séparée en deux équations de manière telle que les équations (1) à (3) s'écrivent, compte tenu des relations (4) et (5), hors des sources :

$$
\begin{aligned}
& \partial_{\mathrm{ct}} \tau-(\rho \mathrm{c} / \gamma \beta) \operatorname{div} \mathbf{v}_{l}=\beta^{-1} \partial_{\mathrm{ct}} \mathrm{p}, \\
& \left(\partial_{\mathrm{ct}}-l_{\mathrm{h}} \Delta\right) \tau=[(\gamma-1) /(\gamma \beta)] \partial_{\mathrm{ct}} \mathrm{p}, \\
& \left(\partial_{\mathrm{ct}}-l_{\mathrm{v}} \Delta\right) \mathbf{v}_{l}=-(\rho c)^{-1} \operatorname{grad} \mathrm{p}, \\
& \left(\partial_{\mathrm{ct}}-l_{\mathrm{v}} \Delta\right) \mathbf{v}_{\mathrm{v}}=\mathbf{0}, \\
& \operatorname{div} \mathbf{v}_{\mathrm{v}}=0,
\end{aligned}
$$


$\operatorname{rot} \mathbf{v} l=\mathbf{0}$,

où les longueurs caractéristiques $l_{\mathrm{v}}, l_{\mathrm{v}}$ et $l_{\mathrm{h}}$ répondent aux définitions suivantes (c désigne la vitesse du son) :

$$
l_{\mathrm{v}}=(\eta+4 \mu / 3) /(\rho c), l_{\mathrm{v}}=\mu /(\rho c), l_{\mathrm{h}}=1 /\left(\rho c \mathrm{C}_{\mathrm{p}}\right) .
$$

La résolution de ces équations passe par celle des équations de propagation, pour $p, \tau$ et $\mathbf{v} l$, équivalentes aux équations (9) à (11). On peut démontrer [22,23] que ces quantités satisfont toutes aux mêmes équations de propagation. A titre d'exemple, la température $\tau$ peut être écrite comme la somme d'une température acoustique $\tau_{\mathfrak{a}}$ et d'une température entropique $\tau_{\mathrm{h}}$, solutions respectives des équations homogènes:

où

$$
\begin{aligned}
& {\left[\left(\partial_{\mathrm{ct}}\right)^{2}-(\Gamma+\mathrm{R}) \Delta\right] \tau_{\mathrm{a}}=0 \quad, \quad\left[\left(\partial_{\mathrm{ct}}\right)^{2}-(\Gamma-\mathrm{R}) \Delta\right] \tau_{\mathrm{h}}=0,} \\
& 2 \Gamma=1+\left(l_{\mathrm{v}}+\gamma l_{\mathrm{h}}\right) \partial_{\mathrm{ct}}, \\
& 2 \mathrm{R}=\left\{1+2\left[l_{\mathrm{v}}-(2-\gamma) l_{\mathrm{h}}\right] \partial_{\mathrm{ct}}+\left(l_{\mathrm{v}}-\gamma l_{\mathrm{h}}\right)^{2}\left(\partial_{\mathrm{ct}}\right)^{2}\right\}^{1 / 2} .
\end{aligned}
$$

A noter que l'équation (16.b) est une équation de diffusion car le développement de Taylor de la fonction $(\Gamma-R)$ montre que l'opérateur $\partial_{c t}$ peut être factorisé $: \tau_{h}$ est associé au transfert thermique dû à la conduction du même nom.

Dans le domaine fréquentiel ( $\left.\partial_{c t}=\mathrm{ik}\right)$, les équations (16.a-b) deviennent :

où

$$
\left(\Delta+\mathrm{k}_{\mathrm{a}}^{2}\right) \tau_{\mathrm{a}}=0 \quad, \quad\left(\Delta+\mathrm{k}_{\mathrm{h}}^{2}\right) \tau_{\mathrm{h}}=0
$$

$$
\mathrm{k}_{\mathrm{a}}^{2}=\mathrm{k}^{2}\left(1+\mathrm{ik} l_{\mathrm{vh}}-\mathrm{k}^{2} l_{\mathrm{vh}} l_{\mathrm{h}}\right)^{-1} \text {, }
$$

$\mathrm{k}_{\mathrm{h}}{ }^{2}=-\mathrm{ik}\left[l_{\mathrm{h}}\left(1-\mathrm{ik} l_{\mathrm{vh}}\right)\right]^{-1}$,

avec $\quad l_{\mathrm{vh}}=l_{\mathrm{v}}+(\gamma-1) l_{\mathrm{h}} \quad, \quad l_{\mathrm{vh}}=(\gamma-1)\left(l_{\mathrm{h}}-l_{\mathrm{v}}\right)$.

A noter que l'équation (12) s'écrit, dans le domaine fréquentiel :

$$
\begin{array}{ll} 
& \left(\Delta+\mathrm{k}_{\mathrm{v}}^{2}\right) \mathbf{v}_{\mathrm{v}}=\mathbf{0}, \\
\text { où } & \mathrm{k}_{\mathrm{v}}^{2}=-\mathrm{ik} / l_{\mathrm{v}}^{\prime},
\end{array}
$$

et les équations (17) et (18) donnent les expressions exactes des nombres d'onde $\mathrm{k}_{\mathrm{a}}, \mathrm{k}_{\mathrm{h}}$ et $\mathrm{k}_{\mathrm{V}}$, associés respectivement au mouvement acoustique, au mouvement entropique lié à la conduction thermique et au mouvement tourbillonnaire lié â la viscosité (ces résultats furent obtenus en 1989 [21]).

A partir des équations (9) à (12) il est facile d'obtenir des expressions pour la variation de pression $p$ et pour la vitesse laminaire $\mathbf{v} l$ en fonction de la température $\tau=\tau_{a}+\tau_{h}\left(\tau_{a}\right.$ et $\tau_{h}$ étant solutions des équations $17 . \mathrm{a}-\mathrm{b})$; dans le domaine fréquentiel ces expressions s'écrivent:

$$
\begin{aligned}
& \mathrm{p}=[\gamma \beta /(\gamma-1)]\left(\xi_{\mathrm{ha}} \tau_{\mathrm{a}}+\xi_{\mathrm{hh}} \tau_{\mathrm{h}}\right), \\
& \mathbf{v} l=(\mathrm{i} / \rho \omega)[\gamma \beta /(\gamma-1)]\left(\xi_{\mathrm{a}} \operatorname{grad} \tau_{\mathrm{a}}+\xi_{\mathrm{h}} \operatorname{grad} \tau_{\mathrm{h}}\right),
\end{aligned}
$$

avec $\quad \xi_{\mu v}=1-\mathrm{i} l_{\mu} \mathrm{k}_{v}{ }^{2 / \mathrm{k}}$ and $\xi_{v}=\xi_{\mathrm{h} v} / \xi_{\mathrm{v} v}$, où l'indice " $\mu$ " est mis pour " $h$ " ou" $v$ " et où l'indice " $v$ " est mis pour "a" ou " $h$ ", les indices "a,h, $\mathrm{v}$ " correspondant aux trois sortes de mode (acoustique, entropique et tourbillonnaire) .

\subsubsection{Conditions aux frontières, applications [23].}

Dans la plupart des applications, la conductivité thermique et la capacité calorifique des matériaux qui constituent les parois limites du domaine considéré ont des valeurs très supérieures à leurs homologues pour le fluide. Par suite, négligeant le transfert thermique parallèle à la paroi (la température varie lentement dans cette direction) et le très faible saut de température en paroi [24], l'équation de continuité du flux de chaleur à l'interface revient à écrire que la température totale $\mathrm{T}$ reste constante sur la paroi, soit:

$$
\tau_{\mathrm{a}}+\tau_{\mathrm{h}}=0 \quad \text { sur la frontière } \text {. }
$$

De plus, pour des parois parfaitement rigides, si la très faible vitesse de glissement à l'interface est négligée [24], la vitesse particulaire totale $\mathbf{v}$ est supposée nulle en frontière:

$$
\begin{array}{ll}
v_{\mathrm{au}}+v_{\mathrm{hu}}+v_{\mathrm{vu}}=0 & \text { sur la frontière, } \\
\mathbf{v}_{\mathrm{aw}}+\mathbf{v}_{\mathrm{hw}}+\mathbf{v}_{\mathbf{v w}}=0 & \text { sur la frontière, }
\end{array}
$$

où l'équation (22) représente la composante de la vitesse normale à la paroi et l'équation (23) sa composante tangentielle (la somme de la vitesse acoustique $\mathbf{v}_{\mathbf{a}}$ et de la vitesse entropique $\mathbf{v}_{\mathbf{h}}$ est la vitesse laminaire $\mathbf{v}_{l}$ introduite dans l'équation (8)) . 
Chaque quantité est exprimée dans la suite sous la forme du produit d'une fonction de " $u$ ", coordonnée locale normale à la frontière, et d'une fonction de $\mathbf{w}$, coordonnées dans le plan tangent, attribuant le même nom à la première fonction (fonction de u) qu'à la quantité considérée elle-même; plus précisément on remplace désormais par exemple $\tau_{\mathrm{a}}$ par $\tau_{\mathrm{a}}(\mathrm{u}) \psi(\mathbf{w})$ que l'on écrit plus simplement $\tau_{\mathrm{a}} \psi$. Reportant alors l'expression (20) de $\mathbf{v}_{l}$ dans les conditions aux frontières (21) à (23), et écrivant que ces conditions doivent être satisfaites quelquesoit $\mathbf{w}$ sur la frontière, une équation de dispersion nouvelle est obtenue [23] :

$$
\left(1-\zeta_{h} / \zeta_{\mathrm{a}}\right) v_{v u} / v_{v w}=\left(\partial_{u} \tau_{\mathrm{a}}\right) / \tau_{\mathrm{a}}-\left(\partial_{\mathrm{u}} \tau_{\mathrm{h}}\right) / \tau_{\mathrm{h}},
$$

où l'indice " $w$ " représente une quelconque des deux composantes du vecteur "w" .

Cette dernière équation donne lieu à des résultats utiles que nous présentons ci-dessous, en coordonnées cartésiennes, cylindriques et sphériques.

En coordonnées cartésiennes, considérons un milieu semi-infini limité par un plan rigide infini situé en $\mathrm{x}=0$. Les effets des modes thermique et de cisaillement à l'interface $\mathrm{x}=0$ peuvent être traités en faisant usage du concept d'admittance spécifique de frontière $Y_{a}=\rho c v_{a} / p_{a}$, dont l'expression suivante peut être déduite simplement de l'équation de dispersion (24) au terme d'un calcul approché:

$$
\mathrm{Y}_{\mathrm{a}}=(\mathrm{ik})^{1 / 2}\left\{\left[1-\left(\mathrm{k}_{\mathrm{ax}} / \mathrm{k}_{\mathrm{a}}\right)^{2}\right]\left(l_{\mathrm{v}}\right)^{1 / 2}+(\gamma-1)\left(l_{\mathrm{h}}\right)^{1 / 2}\right\}
$$

Ce résultat (mentionné au $\S 2.2$ ci-dessus) fut trouvé par L. Cremer en 1948, par une autre voie.

En coordonnées cylindriques, l'équation de dispersion (24) n'est autre que l'équation exacte qui permet d'obtenir le nombre d'onde axial complexe pour toutes les formes de modes (propagatifs ou évanescents) dans un guide cylindrique (résultat obtenu en 1988 [23]). Cette équation s'écrit:

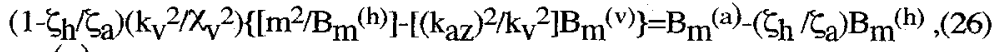

avec

$$
\mathrm{B}_{\mathrm{m}}(\mu)=\chi_{\mu} \mathrm{R} \mathrm{J}_{\mathrm{m}}\left(\chi_{\mu} \mathrm{R}\right) / \mathrm{J}_{\mathrm{m}}\left(\chi_{\mu} \mathrm{R}\right)
$$

où $\mathrm{J}_{\mathrm{m}}$ est la fonction de Bessel cylindrique du $\mathrm{m}^{\text {ième }}$ ordre, $\mathrm{R}$ le rayon du tube, et

$$
\chi_{\mu}=\left[k_{\mu}{ }^{2}-\left(k_{a z}\right)^{2}\right]^{1 / 2}
$$

Cette équation exacte a été utilisée en 1988 [15] pour généraliser l'expression obtenue en 1949 [25] pour le nombre d'onde axial.

En coordonnées sphériques, faisant usage des solutions qui font intervenir les fonctions de Bessel sphériques, l'équation de dispersion (24) prend la forme ci-dessous (qui permet de déterminer les fréquences de résonance d'un fluide contenu dans une sphère):

$$
\left(1-\zeta_{h} / \zeta_{a}\right) n(n+1) /\left(1+b_{m}{ }^{(v)}\right)=b_{m^{(a)}}-\left(\zeta_{h} / \zeta_{a}\right) b_{m}{ }^{(h)}
$$

avec $\quad b_{m}{ }^{(\mu)}=\left[k_{\mu} R j^{\prime}{ }_{n}\left(k_{\mu} R\right)\right] / j_{n}\left(k_{\mu} R\right)$, R représentant le rayon de la sphère .

Ce résultat fut publié en 1988 [17].

\section{APPLICATIONS RECENTES: CHAMPS ACOUSTIQUES EN CAVITES.}

Les équations présentées dans les paragraphes précédents sont utilisées pour résoudre certains problèmes pratiques impliquant des guides ou des cavités. Nous nous intéressons plus particulièrement ici aux applications utilisant des fluides en cavités, qui nécessitent une bonne connaissance de la structure spatiale des champs acoustiques (couplages de modes, effets de sources locales et non locales,...), et qui nécessitent également des résultats précis pour les fréquences de résonances, les facteurs de qualité, l'effet de Coriolis, etc. Enfin, nous abordons les problèmes nouveaux qui apparaissent dans l'étude des champs en cavités miniatures (gravure silicium).

\subsection{Résonateur sphérique pour la mesure précise de propriétés de gaz.}

La première application que nous mentionnons ici très brièvement a pour objet l'obtention de propriétés thermophysiques de gaz, spécialement à "basse" température et "haute" pression, à partir de la mesure des fréquences de résonances acoustiques d'un résonnateur sphérique (diamètre $12 \mathrm{~cm}$, défaut de sphéricité inférieur à $10 \mu \mathrm{m}$ ) [17,26]. La température est contrôlée avec une précision de $0,001 \mathrm{~K}$ (la précision de sa mesure n'excédant pas $0,01 \mathrm{~K}$ ); le domaine de fréquence couvert va de 1 à $40 \mathrm{kHz}$. Le facteur de qualité élevé (entre 1000 et 10000 ) autorise 
la mesure précise des fréquences de résonances, en faisant usage d'un modèle détaillé qui reproduit l'allure de la courbe de résonance. Le rapport de la vitesse du son dans le gaz et du rayon du résonateur peut alors être mesuré avec une précision de l'ordre de $10^{-5}$ (dans le domaine 1 à 200 bars et 200 à $320 \mathrm{~K}$ du plan $\mathrm{P}, \mathrm{T})$.

\subsection{Couplages de modes en cavité.}

Dans ce paragraphe nous présentons une méthode adaptée au calcul du couplage de modes acoustiques de fluides en cavités, qui prend en compte les inévitables imperfections de parois (haut-parleurs et microphones à la surface des parois, imperfections de forme, ...), et les effets d'inertie liés aux mouvements imposés au système. Seules les cavités de forme simple (rectangulaire, cylindrique ou sphérique) sont considérées, de manière à conduire à des solutions analytiques. Le couplage de Coriolis et les autres effets d'inertie sont considérés comme des sources de volume, mais il se trouve que les coefficients de couplage qui leur sont liés agissent de façon analogue à ceux qui ont une origine géométrique (ou qui sont liés aux effets des couches limites).

Comme nous l'avons mentionné au paragraphe 2.3 , pour les cavités dont les dimensions sont grandes en comparaison des épaisseurs des couches limites visqueuses et thermiques, c'està-dire nettement supérieures à $10 \mu \mathrm{m}$, le mouvement acoustique prédomine dans tout le volume (hors des couches limites), et par suite le comportement acoustique peut être décrit dans le domaine fréquentiel par l'équation de Helmholtz en fluide dissipatif (17-a), dans laquelle le nombre d'onde acoustique $\mathrm{k}_{\mathrm{a}}$ est donné par l'équation (17-c):

$$
\left(\Delta+\mathrm{k}_{\mathrm{a}}{ }^{2}\right) \mathrm{pa}_{\mathrm{a}}=\rho \operatorname{divF} \text {, dans le domaine (D) de la cavité, }
$$

où $\mathbf{F}$ représente les forces "extérieures" appliquées au fluide ( source d'énergie et forces inertielles).

En raison des conditions à imposer sur les frontières de la cavité, les trois modes (acoustique, entropique et tourbillonnaire) interagissent fortement à l'intérieur des couches limites : le mode entropique et le mode tourbillonnaire sont générés par la réaction des parois en présence du champ acoustique, générant par retour une petite composante du champ acoustique sur la frontière. Ces phénomènes peuvent être traduits par la condition en frontière de type impédance (équation 25), qu'il reste à combiner avec les impédances équivalentes aux différentes imperfections de parois mentionnées ci-dessus pour exprimer les conditions aux frontières du problème.

La solution d'un tel problème peut être recherchée sous forme de développement sur une base de fonctions propres $\psi_{\mathrm{N}}$ adaptée, qui prend éventuellement en compte certaines perturbations de frontière:

$$
\mathrm{p}_{\mathrm{a}}(\mathbf{r})=\Sigma_{\mathrm{N}} A_{\mathrm{N}} \psi_{\mathrm{N}}(\mathbf{r})
$$

en faisant usage de l'équation intégrale classique suivante:

$$
\mathrm{p}_{\mathrm{a}}(\mathbf{\mathbf { r }})=\iiint_{\mathrm{D}} \mathrm{G}\left(\mathbf{r}, \mathbf{r}^{\prime}\right) \rho \operatorname{div} \mathbf{F} \mathrm{d} \mathbf{r}^{\prime}+\iint_{\partial \mathrm{D}}\left[\mathrm{G}\left(\mathbf{r}, \mathbf{r}^{\prime}\right) \partial_{\mathrm{n}} \mathrm{p}_{\mathrm{a}}\left(\mathbf{r}^{\prime}\right)+\mathrm{p}_{\mathrm{a}}\left(\mathbf{r}^{\prime}\right) \partial_{\mathrm{n}}, \mathrm{G}\left(\mathbf{r}, \mathbf{r}^{\prime}\right)\right] \mathrm{d} \mathbf{r}^{\prime},
$$

où la fonction de Green $G\left(\mathbf{r}, \mathbf{r}^{\prime}\right)$ est choisie de manière telle qu'elle satisfasse aux mêmes conditions aux frontières que les fonctions propres utilisées. Fonctions propres et valeurs propres sont complexes: les coefficients des développements ne comportent de ce fait pas de pôles. Formulé de la sorte, le problème se transforme en un système d'équations algébriques:

$$
[(D)+(\alpha)](A)+(S)=0
$$

où (A) est la matrice colonne des inconnues $A_{N}$,

(S) est la matrice colonne qui représente le transfert d'énergie entre sources et modes propres, le Nième terme étant $S_{\mathrm{N}}=\iiint_{D} \psi_{\mathrm{N}} \rho \operatorname{div} \mathbf{F} \mathrm{d} \mathbf{r}$,

(D) est la matrice diagonale des coefficients $\left(\mathrm{k}_{N}{ }^{2}-\mathrm{k}_{\mathrm{a}}{ }^{2}\right)$ qui met en évidence les résonances,

$(\alpha)$ est la matrice non diagonale définie par ses éléments $\alpha_{M N}=\iint_{\partial D} \psi_{N}[i k Y] \psi_{M} d r$ qui introduit les couplages de modes dus aux diverses perturbations .

Dans le cadre de l'approximation linéaire, lorsque le système est mis en rotation, l'effet de Coriolis est le seul effet d'inertie à prendre en considération (on peut montrer que les autres facteurs d'inertie n'ont pas d'effet acoustique). Le terme de Coriolis $\left(\rho \operatorname{div} F_{c}\right)$, inclus dans le membre de droite de l'équation (28), peut être successivement écrit:

$$
\rho \operatorname{div}(2 \Omega \Lambda v)=2 \rho[v \cdot \operatorname{rot} \Omega-\Omega \cdot \operatorname{rotv}]=-2 \rho \Omega \cdot \operatorname{rotv}=-2 \rho \Omega \cdot \operatorname{rotv}_{\mathrm{V}} \text {, }
$$


où $\Omega$ est le vecteur vitesse de rotation de la cavité, et où $\mathbf{v}$ et $\mathbf{v}_{\mathrm{V}}$ désignent respectivement la vitesse particulaire totale et la vitesse tourbillonnaire associée.

On constate que le seul effet d'inertie qui agit sur le champ acoustique dans la cavité est l'effet de l'accélération de Coriolis sur la composante tourbillonnaire du champ de vitesse interne, qui est très largement prépondérant près des parois, à l'intérieur des couches limites. Par suite, compte tenu de la solution au voisinage de la paroi de l'équation de diffusion (12) pour la vitesse tourbillonnaire $\mathbf{v}_{\mathrm{y}}$ et de la condition (14), l'effet de Coriolis est pris en compte dans la matrice non diagonale $(\alpha)$ en y ajoutant des éléments de la forme [27,28]:

$$
\alpha_{\mathrm{MN}}=2(\mathrm{i} \omega)^{-1} \iint_{\partial \mathrm{D}} \psi_{\mathrm{M}} \Omega .\left(\mathbf{u} \Lambda \operatorname{grad}_{\mathrm{T}} \psi_{\mathrm{N}}\right) \mathrm{d} \mathbf{r},
$$

où $\operatorname{grad}_{\mathrm{T}}$ représente les composantes de l'opérateur grad dans le plan tangent à la frontière et $\mathbf{u}$ une coordonnée normale à la paroi (sortante) dont l'origine est sur la paroi.

Ce résultat montre que l'effet de Coriolis sur le champ acoustique se traduit par un couplage de modes qui prend son origine dans les couches limites tourbillonnaires aux parois, et qui est pris en compte dans un terme de couplage en surface (comme ceux qui proviennent des autres origines mentionnées plus haut).

Les résultats théoriques et expérimentaux obtenus sur des gyromètres acoustiques, dont le fonctionnement repose sur les phénomènes décrits précédemment, montrent que l'on peut atteindre une sensibilité meilleure que $0,01 \%$ s (la vitesse angulaire de l'aiguille des minutes d'une montre est dix fois supérieure), et que le système fonctionne encore pour des vitesses très élevées.

Les avantages du gyromètre acoustique sur les gyromètres à toupie (par exemple) sont les suivants: une plus faible consommation électrique, une durée de vie quasi-illimitée, une faible sensibilité à la température et un coût beaucoup plus faible [29]. De plus, dans la mesure où hautparleurs et microphones peuvent être miniaturisés, ce type de gyromètre peut devenir très peu encombrant. Les technologies modernes de gravure sur silicium autorisent aujourd'hui l'ultraminiaturisation des transducteurs (et donc du gyromètre acoustique), et ouvrent corrélativement la voie à un nouveau champ d'investigation dont l'état actuel est présenté dans le paragraphe qui suit.

\subsection{Transducteurs et cavités miniatures $[30,34]$.}

Depuis une décennie environ, la demande croissante de transducteurs miniatures et le large usage des technologies sur silicium, ont permis la réalisation de microphones à condensateur dont l'apparition sur le marché est attendue dans les années à venir, et permettent la réalisation de haut-parleurs miniatures (et de cavités en vue d'applications à la gyrométrie notamment). Les modèles théoriques en usage aujourd'hui sont ceux qui existent pour les microphones et les haut-parleurs (voire les cavités) conventionnels ; mais le besoin de modèles précis pour les champs acoustiques dans de tels systèmes, de plus en plus petits, apparait aujourd'hui. Le paramètre clé dans ces systèmes n'est autre que l'épaisseur des couches de fluide entre diaphragme et électrode, qui devient du même ordre de grandeur que l'épaisseur des couches limites visqueuses et thermiques.

Les modèles disponibles dans la littérature prennent en compte l'inertie, la compressibilité et la viscosité de cisaillement du fluide. Mais ils admettent diverses hypothèses : la vitesse tourbillonnaire n'est pas complètement prise en compte et la viscosité de volume est négligée, la conduction thermique est le plus souvent supposée nulle et la vitesse laminaire associée est toujours négligée, le champ de pression suivant la normale à la membrane est supposé uniforme et la composante suivant cette normale de la vitesse particulaire nulle, la condition à la frontière périphérique pour la pression dans le fluide est souvent celle de Dirichlet, alors même que ce dernier est en espace clos, etc .

Aussi est-il nécessaire aujourd'hui de proposer un modèle théorique qui n'admet plus de telles hypothèses, qui ne sont plus acceptables en raison de l'importance relative prise par les couches limites visqueuses et thermiques. Le système d'équations linéarisées sur lequel repose l'étude comprend pour l'essentiel l'équation de Stokes-Navier, l'équation de conservation de la masse et l'équation de conduction de la chaleur [équations (1) à (5)], équations couplées à celle régissant le mouvement de la membrane. Les conditions aux frontières retenues sont celles d'usage courant sur les parois solides - variation de température nulle, continuité des composantes normales et tangentielles des vitesses particulaires totales aux interfaces fluide-solide -, mais sont 
de type impédance associée à une loi polytropique à l'interface entre le film de fluide et un volume d'expansion .

Une solution générale de ce problème, qui n'admet plus les approximations d'usage rappelées ci-dessus, ne fait qu'apparaitre aujourd'hui dans la littérature [35,36,37]. Cette solution, qui permet d'accéder à une description précise de microphones (et haut-parleurs) miniaturisés, est particulièrement utile pour l'étude de transducteurs fonctionnant au delà de l'audible (proche ultrasonore, jusqu'à $100 \mathrm{kHz}$ ). Dans le domaine des basses fréquences, de nouveaux modèles à constantes localisées peuvent être obtenus à partir des résultats mentionnés ci-dessus, en écrivant que les dimensions du système sont petites par rapport à la longueur d'onde. En hautes fréquences, des modèles plus sophistiqués doivent pouvoir décrire les effets des phénomènes tourbillonnaires et entropiques qui jouent un rôle dont l'importance va croissante avec la fréquence. Ces travaux sont actuellement en cours...

\section{REFERENCES}

[1] F OLSON H., Dynamical Analogies (Van Nostrand, New-York, 1948).

[2] MERHAUT J., Theory of Electroacoustics, (Mc Graw-Hill, New-York, 1981).

[3] BERANEK L.L., Acoustics, (Mc Graw Hill, New-York, 1954).

[4] ROSSI M., Electroacoustique, Traité d'Electricité de l'Ecole Polytechnique Fédérale de Lausanne, Vol.XXI, (Presses Polytechniques Romandes, 1986).

[5] SKVOR Z., Vibrating Systems and their Equivalent Circuits, (Elsevier Science Publishers, Amsterdam, Oxford, New-York, Tokyo, 1991).

[6] WONG G.S.K. and EMBLETON T.F.W.,"Arrangement for Precision Reciprocity Calibration of Condenser Microphones", J. Acoust. Soc. Am. 66 (1979) 1275-1280.

[7] KIRCHHOFF G.R., "Ueber die Einfluss der Warmeleitung in einem Gase auf die Schallbewegung", Annalen der Physik Leipzig 134 (1868) 177-193 . English Translation (1974) in: R.B. LINDSAY,ed.,Physical Acoustics, Dowden, Hutchinson and Ross, Stroudsburg.

[8] RAYLEIGH J.W.S., Theory of Sound, (2nd ed.1896, reprinted by Dover, New-York, 1945).

[9] BHATIA A.B., Ultrasonic Absorption, An Introduction to the Theory of Sound Absorption and Dispertion in Gases, Liquids and Solids, (Dover publication, New-York, 1967).

[10] BAUER H.J., "Influence of Transport Mechanisms on Sound Propagation in Gases", Adv. Mol. Relaxation Processes, 2 (1972) 319.

[11] CREMER L.,"On The Acoustic Boundary Layer Outside a Rigid Wall", Arch. Elektr. Uebertr, 2 (1948) 235.

[12] BEATTY R.E. Jr, "Boundary Layer Attenuation of Higher Order Modes in Rectangular and Circular Tubes", J .Acoust. Soc. Am. 22 (1950) 850-854.

[13] BRUNEAU M., GARING Ch. and LEBLOND H., "Quality Factor and Boundary Layer Attenuation of Lower Order Modes in Acoustic Cavities", J. Francais de Physique 46 (1985) 1079-1085.

[14] BRUNEAU A.M., BRUNEAU M., HERZOG Ph. and KERGOMARD J., "Boundary Layer Attenuation of Higher Order Modes in Waveguides", J. Sound Vib. 119 (1) (1987) 15-27.

[15] KERGOMARD J., BRUNEAU M., BRUNEAU A.M. and HERZOG Ph.,"On the Propagation Constant of Higher Order Modes in Cylindrical Waveguides", J. Sound Vib. 126 (1) (1988) 178-181.

[16] HUDDE H.,"The Propagation Constant in Lossy Circular Tubes Near the Cutoff Frequencies of higher-order modes", J. Acoust. Soc. Am., 83 (4) (1988) 1311-1318.

[17] MOLDOVER M.R., MEHL J.B. and GREENSPAN M.,"Gas-filled Spherical Resonators: Theory and Experiment", J. Acoust. Soc. Am., 79 (1986) 253-272.

[18] MORSE P.M. and INGARD K.U., Theoretical Acoustics (Mc Graw-Hill, New-York 1968).

[19] PIERCE A.D., Acoustics: An Introduction to its Physical Principles and Applications (Mc Graw-Hill,New-York, 1981).

[20] BRUNEAU M., Introduction aux Théories de l'Acoustique (Université du Maine éditeur, Le Mans France, 1983).

[21] LEFEBVRE J.P.,"Petites Perturbations d'un Fluide Visqueux Conducteur de la Chaleur dans un Etat Initial Quasi-stationnaire et Quasi-uniforme. Operateur de Diffusion-propagation et Application à l'Acoustique Linéaire", Revue du Cethedec,51 (1977) 103-120.

[22] TRUESDELL C., "Precise Theory of the Absorption and Dispersion of Forced Plane Infinitesimal Waves According to the Navier-Stokes Equation", J. Rat. Mech. Anal. 2 (1953) 643-740. 
[23] BRUNEAU M., HERZOG Ph., KERGOMARD J. and POLACK J.D.,"General Formulation of the Dispersion Equation in Bounded Visco-thermal Fluids, and Application to some Simple Geometries", Wave Motion 11 (1989) 441-451.

[24] RATHNAM K.,"Influence of Velocity Slip and Temperature Jump in Rarefied Gas Acoustic Oscillations in Cylindrical Tubes", J. Sound Vib. 103 (3) (1985) 448-452.

[25] ZWIKKER C., KOSTEN C., Sound Absorbing Materials (Elsevier, Amsterdam, 1949).

[26] BRUNEAU M., COSNARD C., AUDIBERT S. and FAVEAU F.M., "Measurement of the Speed of Sound in Gases inside Resonant Cavities", International Gas Research Congress, Tokyo 1989.

[27] BRUNEAU M. et HERZOG Ph., "Influence des Forces d'Inertie sur les Champs Acoustiques en Cavité", Compte-rendu de l'Académie des Sciences, 307 série II (1988) 719-722. [28] HERZOG Ph. and BRUNEAU M., "Shape Perturbation and Inertial Mode Coupling in Cavities", J. Acoust. Soc. Am., 86 (6) (1989) $2377-2384$.

[29] BRUNEAU M. et LEBLOND H.,"Gyrométrie Acoustique",Revue Scientifique et Technique de la Défense, Paris (1990).

[30] HOHM D. and HESS G., "A Subminiature Condenser Microphone with Silicon Nitride Membrane and Silicon Back-plate", J. Acoust. Soc. Am., 85 (1989) 476-480.

[31] ZUCKERWAR A.J., "Theoretical Response of Condenser Microphones", J. Acoust. Soc. Am., 64 (5) (1978) 1278-1285.

[32] ROBEY D.H., "Theory of the Effect of a Thin Air Film on the Vibrations of a Stretched Circular Membrane", J. Acoust. Soc. Am., 26 (1954) 740-745.

[33] WARREN J.E., BRZEZINSKI A.M. and HAMILTON J.F., "Capacitance Microphone Dynamic Membrane Deflection", J. Acoust. Soc. Am., 54 (5) (1973) 1201-1213.

[34] PETRISKAYA I.G., "Impedance of a Thin Layer of Air in the Harmonic Vibrations of a membrane", Sov. Phys. Acous., 12 (2) (1966) 193-198.

[35] BRUNEAU M., BRUNEAU A.M. and HAMERY P.,"An improved approach to modeling the behaviour of thin fluid film trapped between a vibrating membrane and a backing wall and surrounded by a reservoir at the periphery", Acta Acustica, à paraître (Déc. 1993).

[36] HAMERY P., BRUNEAU M. et BRUNEAU A.M., "Mouvement d'une couche de fluide dissipatif en espace clos sous l'action d'une source étendue", Troisième Congrès Français d'Acoustique, Toulouse France Mai 1994 (Les Editions de Physique, Les Ulis, 1994).

[37] LOTTON P., HUSNIK L., BRUNEAU A.M., BRUNEAUU M. et SKVOR Z., "Modèle à constantes localisées de transducteurs : dissipation dans les couches limites", Troisième Congrès Français d'Acoustique, Toulouse France Mai 1994 (Les Editions de Physique, Les Ulis, 1994). 